\title{
A future prosthetic limb device
}

\author{
C. WILLIAM HALL, M.D. \\ Southwest Research Institute, San Antonio, Texas 78284
}

\section{INTRODUCTION}

The first amputees were undoubtedly survivors of accidental amputations. Similarly, the first adaptive device was probably a forked stick used as a crutch to substitute for the lost function of an amputated leg. The eventual invention of a padded peg leg was a major technical breakthrough and became common practice for several centuries. There are historical accounts of armor manufacturers designing marvelously ingenious artificial joints as early as the 15th and 16th centuries (1). It was not until the 20 th century, however, that any significant technological gains were made in prosthetic devices. Today, a wide variety of technically sophisticated prosthetic appliances are available, many of which exhibit amazingly lifelike function and appearance.

As advanced as the new devices are, however, their use by amputees is still severely hampered by problems at the interface between the appliance and the residual limb, which is the same problem that faced the wearer of the peg leg centuries ago. Perhaps the most troublesome problem today is pain associated with the tissues of the stump that must bear the weight of the

* Technical Notes are published in the Journal as a means of exchanging information concerning an investigator's use of a particular scientific instrumentation or procedure, which might further the course of research. While these original notes are subject to peer review and represent an important contribution to the research literature, they lack controlled comparison studies and are thus different from "scientific articles."

This work was supported by Veterans Administration contract V101(134) P-341. body as it loads the prosthesis. Lower-limb amputees fitted with a good socket can often walk great distances before experiencing pain. However, eventually the stump becomes painful, causing the wearer to limp, which puts unusual stresses on the rest of the musculoskeletal system and leads to general body fatigue. Soft tissue pressure pain could be eliminated if the body's weight were to be transmitted directly from the skeletal system to the prosthesis, rather than through intervening soft tissue.

\section{PERCUTANEOUS LOAD-BEARING SKELETAL EXTENSIONS}

For some years, a small group of researchers in the United States, Canada, and Germany have been developing methods for permanently affixing artificial limbs directly to the skeletal member of the amputated residual limb. The only clinical trials attempted were done by Dutmmer in Germany and Mooney in the United States. Murphy (3) has published an excellent review of the early developments of devices that $I$ shall call percutaneous load-bearing skeletal extensions (PLSE). There are several theoretical advantages of the PLSE that substantiate the need for further development: (1) a more atable attachment, since the "false joint" at the socket is eliminated; 2) a positive swing-hrough and more natural proprioception due to the stable extension of the long bone: 3 ) the elimination of pressure pain and skin deterioration; and 4 ) the potential to bring a PLSE under the direet control of existing orthotopic muscle groups. 


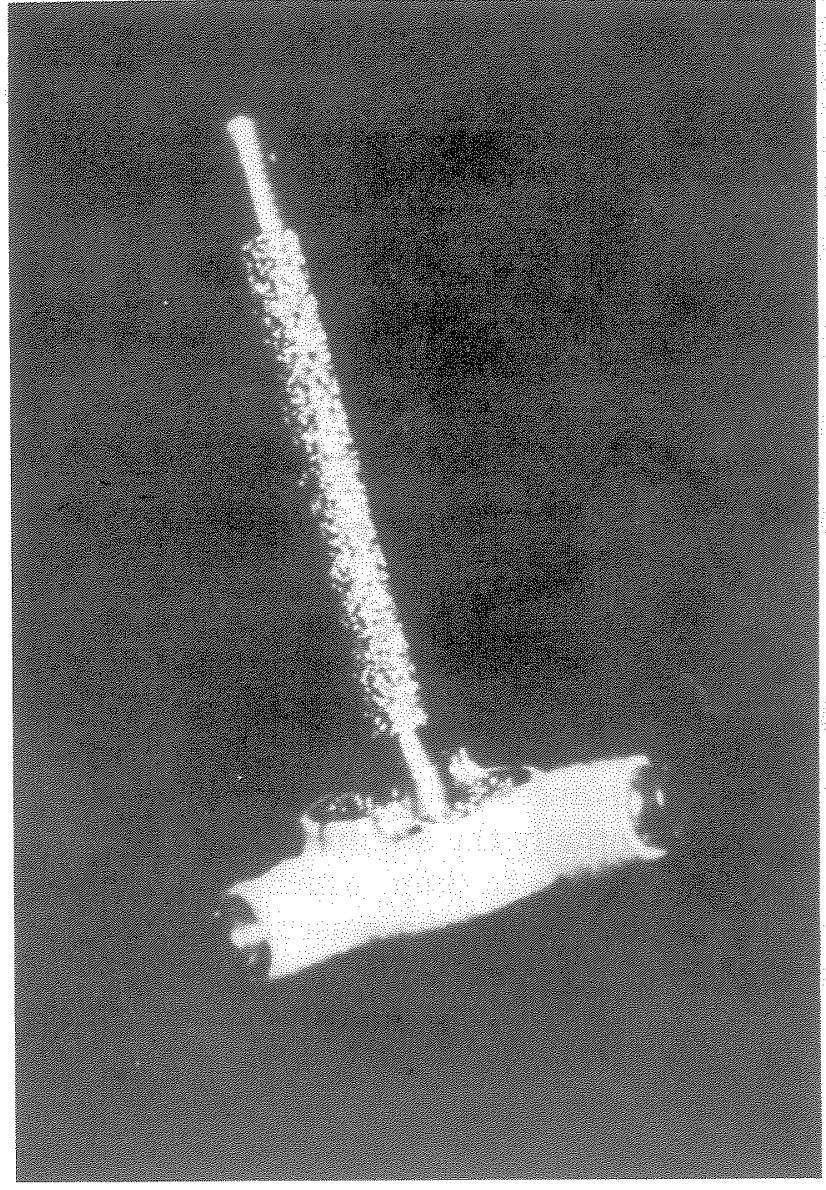

FIGURE 1

Percutaneous load-bearing skeletal extension (PLSE) used currently in this laboratory. Intramedullary shaft and morticed pedestal are covered with a sintered titanium mesh alloy (T, $6 \mathrm{Al}, 4 \mathrm{Va}$ ) where device interfaces with bone. Lugs and pedestal are covered with nylon velour where device interfaces with soft tissue and penetrates the integument.
The characteristic developmental problem facing PLSE research is the identification of the appropriate biomaterial. This biomaterial must be sufficiently strong to support all naturally applied loads, must be mechanically stable and biologically compatible with surrounding tissues, and must form a strong interfacial bond with the skin at the site of penetration. Most of the problems have been solved, but the percutaneous exit site remains the focus of additional research. When the integument fails to remain intact, channels for microbial invasion are formed, resulting in a septic disaster. Except for this single remaining research hurdle, the PLSE would now be an acceptable clinical reality.

Years of experimental frustration have led investigators to accept the fact that penetration of the skin at the end of an amputated stump will always fail because prevalent biaxial stresses tend to enlarge the exit site and tear the interfacial bond. Early clinical trials of permanently attached devices undoubtedly failed for this reason. Recently, guarded claims of success have been offered as a result of using a device that penetrates the skin at right angles to the long axis of the stump where the attendant stresses are more uniaxial and manageable (2). These preliminary indications of success have helped reduce the problem to that of optimum material selection. An elusive solution in its own right, the most effective skin-interfacing material to date has been velour fabrics of nylon or Dacron.

\section{FIGURE 2}

Morticed fit between distal end of tibia and pedestal. Skin closure and delayed exposure of lugs allow tissue ingrowth into velour interstices to become established prior to affixing trifureated external pylon.

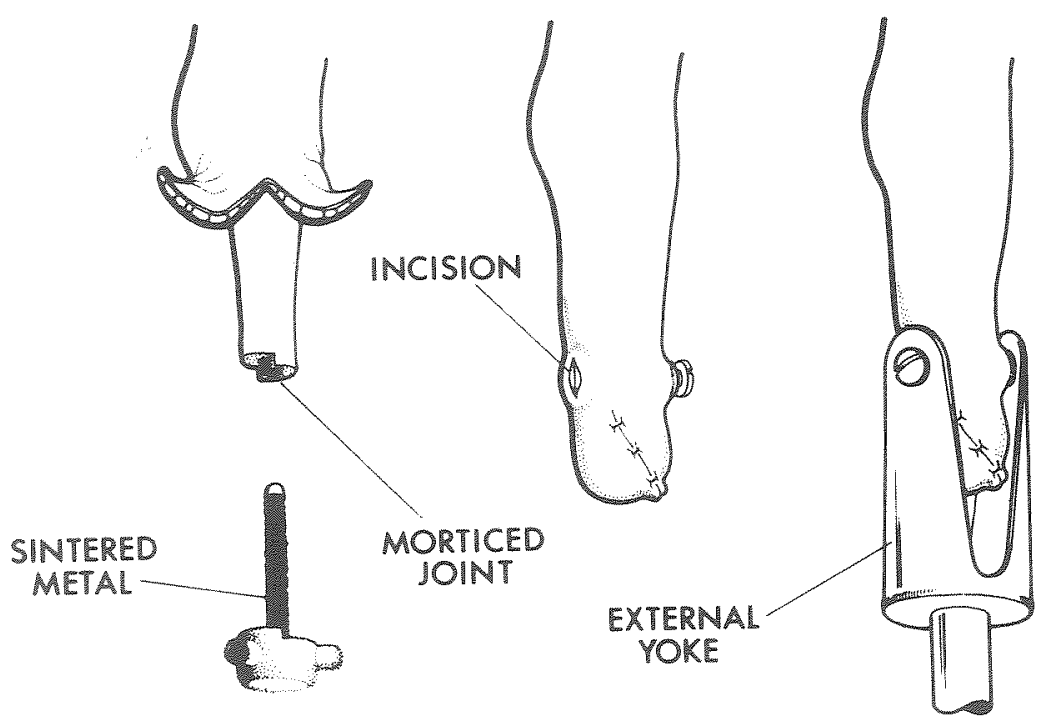




\section{Optimum Selection of Bone-Interfacing Biomaterial}

A solution to the bone interfacing material problem appears more within our grasp. Porous alumina, Bioglass ${ }^{\circledR}$, porous polyethylene, and certain porous metals have all been successfully used. The most stable PLSE bone interface to date used porous sintered titanium mesh. Material choice and configuration are not the final answer, however, because mechanical constraints must also be considered. All bone-interfacing materials will fail unless torque to the intramedullary shaft is controlled. A simple method for torque control has been devised that utilizes the mating of a step in the PLSE pedestal with a morticed step in the long bone end.

The design currently used in this laboratory consists of an intramedullary shaft ending in a step-shaped pedestal with three radiating lugs spaced at 120 degree intervals (Fig. 1). The lugs are the skin-penetrating elements that provide anchorage to the external pylon. Portions of the shaft and pedestal are covered with porous titanium for bone ingrowth, whereas the skin- penetrating elements are covered with velour fabric. Implantation is accomplished by amputating a long bone (usually the tibia), enlarging the intramedullary canal to accept the PLSE shaft, and morticing the distal end to fit the stepped pedestal (Fig. 2). The PLSE is installed with a slight tap of a mallet, and the extensor and flexor tendons are brought over the pedestal and joined by sutures. The wound is closed in layers so as to completely bury the prosthesis. A light cast dressing and a modified Thomas leg splint are then applied, which allows the animal to walk immediately after recovering from anesthesia. After 3 weeks, the dressing and splint are removed and the buried lugs exposed by sharp dissection. A trifureated yoke pylon is then bolted on to the exposed lugs.

Spanish goats have been the animal model of choice (Fig. 3), although a few opportune horses with leg fractures have also been used. One rare pink flamingo from the San Antonio Zoo had a traumatic amputation repaired by having a segment of a carbon fiber fishing rod cemented to the bone and the skin sutured to an adherent velour collar. Canines were used early in the device's



FIGURE: 3

Spanish goat with PLSE cornered in an inspection trap. Ten months after amputation, right hindleg shows a well-healed, noninfected wound. Stance is that of nonamputated animal. 
development, but their incessant gnawing and licking of the wound sites created continual problems. Goats never disturb the wound, and healing is seldom a problem. After the external pylon is bottled to the PLSE, goats usually walk immediately with no apparent limp, pain, or disturbance of gait. After application of an external pylon, the animals are turned out to pasture with no medication or special dietary regimen; they have been allowed to breed and kid and have survived without difficulty for up to 14 months. Inevitably the percutaneous sites become infected due to trauma or due to exposure to manure, mud, and the elements. Animals with infections are sacrificed and the PLSE is retrieved. One goat with a PLSE on a hindlimb jumped a 6-foothigh corral fence and, although periodically seen at distances of several hundred yards, was never recaptured from the wilds of the brush country.

\section{REFERENCES}

1. BIck EM: Sourcebook of Orthopaedics. New York: Hafner, 1968.

2. Drummer G: Personal communication with E. F. Murphy, 1954.

3. HALL CW: Biocompatability of Orthopedic Implants, Vol. II, edited by D. F. Williams. Boca Raton, FL: Chemical Rubber Company Press, 1982, pp. 179-198.
Because of the extreme forces applied to the PLSE by these very active animals, a few of the devices have had the intramedullary metal shaft break at a point near the pedestal attachment.

\section{CONCLUSION}

Since there are many applications for generic percutaneous devices, research efforts to solve the skin-interfacing problem are being carried out in several laboratories throughout the world. This final problem must be resolved before clinical use of the PLSE can be justified. However, it is predictable that implanting a PLSE will someday become a standard orthopedic implant procedure, thereby alleviating some of the difficulties encountered by today's amputees.

4. Mooney V, Predecki PK, Renning J, Gray J: J Mater Res Symp 2 (I): 143-159, 1971.

5. MURPHY FE: History and philosophy of attachment of prostheses to the musculo-skeletal system and of passage through the skin with inert materials. I Biomed Matr Res Symp 4: 275-295, 1973. 